\title{
CÉPHALOPODES, EXPÉRIMENTATION ANIMALE ET LÉGISLATION EUROPÉENNE
}

\author{
CEPHALOPODS, ANIMAL EXPERIMENTATION AND EUROPEAN \\ LEGISLATION
}

Par Laure BONNAUD-PONTICELLI ${ }^{1}$

(Communication présentée le 25 mars 2021, manuscrit accepté le 5 mai 2021)

\section{RÉSUMÉ}

Les céphalopodes sont inclus depuis 2013 dans la législation relative à l'expérimentation animale. L'origine de cette inclusion vient probablement de leurs spécificités neuro-sensorielles (cerveau, œil) et comportementales (mémoire, adaptation) uniques parmi les non-vertébrés. Si le bien-être est maintenant cadré, le référencement clair de la « douleur » et une échelle d'intensité ne sont pas encore disponibles. Des données d'observation sur plusieurs espèces existent cependant. Sans que cela puisse être prouvé scientifiquement (selon le référentiel mammifère), il ne peut être exclu qu'ils ressentent de la douleur, et à ce titre doivent faire l'objet de précautions pour l'éviter. Le chlorure de magnésium $\left(\mathrm{MgCl}_{2}\right)$ et l'éthanol sont des anesthésiques efficaces et peuvent également à fortes doses entrainer la mort. Les méthodes pour euthanasier les céphalopodes ne sont pas décrites dans la législation.

Mots-clés : Céphalopode, Douleur, Expérimentation animale, Législation

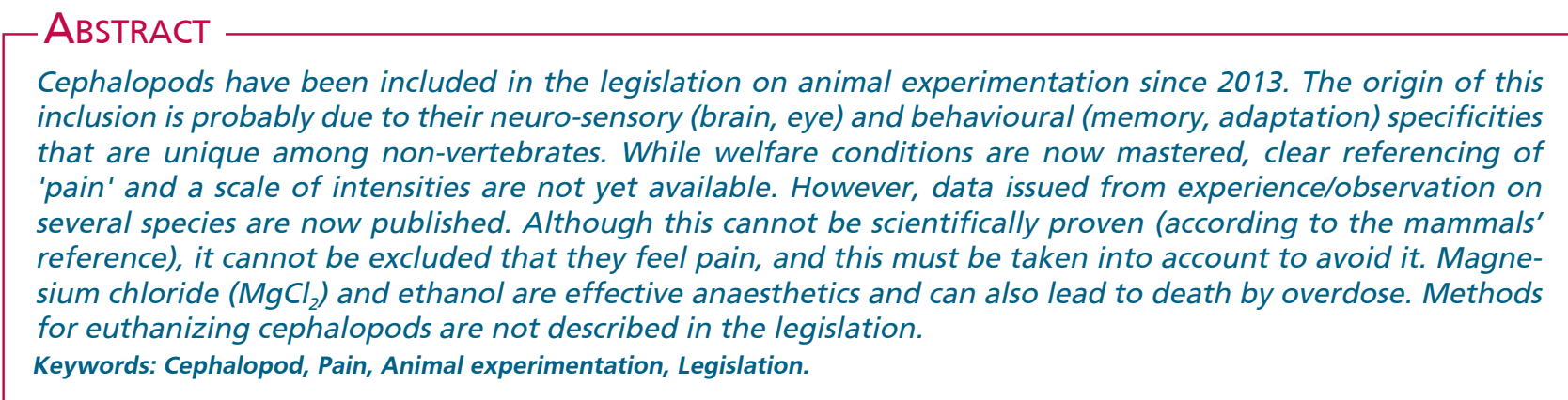

\section{INTRODUCTION}

Par le décret français 2013-118 ajouté à la directive 2010-63-UE de l'union européenne, relative à la protection des animaux utilisés à des fins scientifiques, les céphalopodes sont inclus et les règles utilisées pour les vertébrés doivent désormais s'appliquer à ce groupe de mollusques.

Il est indiqué "Outre les animaux vertébrés, qui comprennent les cyclostomes, les céphalopodes devraient également être inclus dans le champ d'application de la présente directive, car leur aptitude à éprouver de la douleur, de la souffrance, de l'angoisse et un dommage durable est scientifiquement démontrée ».

La législation a quelque peu anticipé les études sur le sujet. Notons qu'en 2013, il n'y avait que très peu d'éléments publiés disponibles sur l'environnement de l'expérimentation. Afin de se conformer à la législation, la mise en place d'un programme

(1) Professeure au Museum National d'Histoire Naturelle. UMR BOREA MNHN-CNRS8067-SU

43 rue Cuvier CP2675005 Paris. Mail : laure.bonnaud@mnhn.fr 
européen COST «FA1301 - A network for improvement of cephalopod welfare and husbandry in research, aquaculture and fisheries (CephsInAction) » a permis le regroupement de données, d'informations et des publications y compris des "guidelines " et des réflexions relatives à la règle des $3 \mathrm{R}$ ont été publiés (Andrews et al. 2013 ; Fiorito et al. 2014, 2015 ; Winlow et Di Cosmo, 2019). Ces travaux constituent une aide indéniable à la fois sur le bien-être animal et l'optimisation des conditions de maintien ou d'élevage des animaux mais également sur la prise en compte de la douleur pour les expérimentations.

Le présent article a pour objet la description des raisons biologiques qui justifient cette inscription, l'état des connaissances sur la physiologie de la douleur, de la souffrance et les conditions d'application chez les céphalopodes. Notons d'emblée qu'il y a une grande différence entre les espèces de céphalopodes, suivant les groupes, leur taille et leur environnement, (tempéré, polaire ou tropical, côtier ou profond), et bien sur, leur âge ou stade du cycle de vie. Par ailleurs, il n'y a pas, dans les recherches actuelles, d'espèce unique de céphalopode modèle qui fasse consensus. Cependant, on peut évaluer à moins d'une dizaine le nombre d'espèces impliquées dans des projets nécessitant de l'expérimentation animale.

\section{ANATOMIE ET COMPORTEMENT}

Parmi les céphalopodes actuels, seuls les Nautiles ont encore une coquille externe; ils ne font pas l'objet d'expérimentation animale mais sont exposés dans des aquariums et figurent à l'annexe II de la CITES. Les autres céphalopodes incluent les seiches, les calmars, les pieuvres, dont de nombreuses espèces ont un intérêt économique et sont abondamment pêchées. Quelques espèces sont actuellement utilisées pour de l'expérimentation animale en Europe, où la règlementation doit être appliquée et hors Europe. Les céphalopodes ont été inclus dans la législation pour leurs comportements, intelligibles d'un point de vue anthropocentrique. L'essentiel des comportements des céphalopodes sont guidés visuellement : les yeux, camérulaires, possèdent une rétine, un cristallin, un iris, voire une cornée (Figure 1). Les informations visuelles sont intégrées dans le système nerveux central. Celui-ci est constitué de 2 lobes optiques, comportant 60 millions de neurones chacun, et d'un cerveau de 50 millions de neurones. Ce cerveau, enfermé dans une capsule cartilagineuse, est divisé en de multiples lobes et hiérarchisé fonctionnellement : les lobes ont des fonctions différentielles, sensorielles ou motrices, et sont reliés par des commissures. Ce système nerveux central permet une analyse et une interprétation des informations visuelles conduisant à des comportements multiformes et adaptés à une situation donnée. A cela s'ajoute le système nerveux périphérique, celui des bras dont l'autonomie (relative) a été montrée, mais aussi les ganglions stellaires et la peau (Figure 1). L'ensemble est à l'origine des nombreux comportements de camouflage, de communication et des capacités d'apprentissage, d'habituation et de mémorisations sus-jacentes, rapportés par de nombreuses études (récemment (Shomrat et al. 2015 ; Zepeda et al. 2017) parmi d'autres).

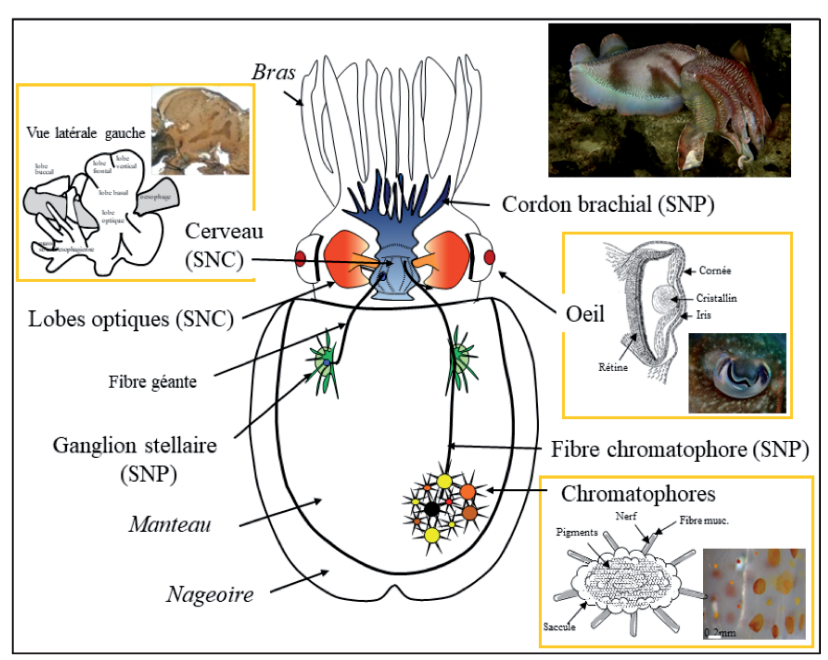

Figure 1 : Morphologie et anatomie d'un céphalopode. Vue de dessus. Exemple de la seiche. Photo en haut à droite : Sepia pharaonis@Mark Norman. Le système nerveux central (SNC) est composé du cerveau (constitué de plusieurs lobes et organisé autour de l'œesophage) et des lobes optiques localisés entre les deux yeux. Les yeux sont composés d'une rétine, d'un cristallin, d'un iris et d'une cornée. Photo : œil de Sepia officinalis@ Yann Bassaglia. Le système nerveux périphérique (SNP) est constitué des nerfs brachiaux, des fibres géantes, des nerfs des chromatophores et des ganglions stellaires. Les chromatophores sont des organes neuro-musculaires responsables de la coloration pigmentaire et des motifs de la peau.

Ces comportements par l'observation, l'utilisation de leurs bras pour effectuer des actions qui nous sont familières " ouvrir un bocal, prendre une photo " et leurs capacités de conscience du corps en particulier (utiliser uniquement le passage correspondant à la distance entre leurs deux yeux par l'analyse visuelle) ont entrainé, de fait, leur classement dans la catégorie des animaux susceptibles de montrer de "l'intelligence ». Parallèlement les motifs colorés changeants qui semblent correspondre à des "émotions " (!) interrogent également leurs capacités de communication mais aussi leurs réactions exacerbées à toute modification de leur environnement : ces particularités ont conduit les expérimentateurs à s'interroger sur leur sensation de souffrance et de douleur, voire d'angoisse...

\section{DOULEUR ET SOUFFRANCE}

L'identification de la douleur (selon le référentiel connu chez les mammifères) et donc de la souffrance est difficile à effectuer chez les céphalopodes. Les indicateurs objectifs de douleur, souffrance ou stress, se sont précisés ces dernières années, mais s'il y a certaines manifestations communes, l'importante diversité de réactions entre les espèces empêche toute généralisation et établissement d'une échelle des réactions reflétant une intensité de stress. Parmi les comportements référencés, il y a 1) des manifestations immédiates évidentes de stress dans le comportement : éjections d'encre répétées, une locomotion erratique et rapide, des motifs changeants et "incohérents ", une ventilation accélérée et 2) des manifestations de douleur et mal-être 
observables sur le court et moyen terme : position anormale des bras, automutilation, disruption de patterns (tête noire, corps blanc ou inversement) ou motifs uniformes pales, flottabilité positive, apathie, non-coordination de la ventilation ; ces derniers éléments signifiant un dysfonctionnement physiologique probablement irréversible.

Il est parfois dit que ces animaux peuvent " mourir de stress ". Par exemple des éjections d'encre répétées entrainent une perte considérable d'énergie en même temps que des auto-lésions de peau et peuvent conduire à la mort de l'animal (Jiang et al. 2019). Il est également fort probable que certains critères ne soient pas visibles mais relèvent de réactions physiologiques. Il convient ainsi de ne pas négliger les "marqueurs de stress".

A côté des comportements, les blessures infligées à l'animal sans précaution entrainent des réactions vives de contraction et de fuite. Ils montrent pour certains, une " mémoire " de la "douleur ", lorsqu'une blessure a été opérée sans analgésique (Crook et al. 2014 ; Crook, 2021). Si la blessure a été faite avec analgésique, l'animal recouvre un comportement normal et après élimination de l'effet analgésiant, ne semble pas ressentir d'effet consécutif (Crook et al. 2014 ; Crook 2021). Par ailleurs, Crook (2021) a montré un effet associatif entre la douleur et l'environnement d'une part et l'application de l'analgésiant et l'environnement d'autre part. Ces caractéristiques constituent des éléments permettant de définir l'existence de douleur chez un animal. Les céphalopodes répondent à plusieurs d'entre eux mais pas tous : la présence de nocicepteurs est probable mais pas certaine, de même que leurs liaisons avec le système nerveux centralisé (présent indéniablement) ; il y a en revanche des récepteurs aux opioïdes même si leur fonction et rôle ne sont pas encore caractérisés.

En résumé, il y a bien des réactions et des comportements relatifs au stress, mais l'absence d'identification claire de nocicepteurs et de données objectives suffisantes ne permet pas à l'heure actuelle d'établir avec certitude qu'ils ressentent de la « douleur " ni d'établir une échelle d'intensité. Cependant, il ne peut pas être exclu qu'il existe un système de nociception conduisant à une sensation. Ils possèdent un certain nombre d'attributs qui suggèrent qu'ils pourraient ressentir de la douleur voire de la souffrance, ce qui implique de prendre les précautions nécessaires pour l'éviter.

\section{ANALGÉSIQUES ET ANESTHÉSIQUES}

De nombreux anesthésiants (comme le MS222-tricaine methanesulfonate) et analgésiques (comme l'eugénol) utilisés chez les poissons ont été testés chez les céphalopodes, comme une évidence du fait de l'environnement aquatique. C'était sans compter sur les spécificités des céphalopodes. Très peu sont efficaces. Connu et testé chez les non vertébrés depuis longtemps, le $\mathrm{MgCl}_{2}$ s'est révélé un analgésique (local) et anesthésique efficace chez les céphalopodes (Crook et al. 2013 ; Butler-Struben et al. 2018). Avec l'éthanol, ils constituent des anesthésiques efficaces à des concentrations variables suivant les espèces et la température. Leurs effets sont réversibles. Une étude récente approfondie sur les réactions (spatiales et temporelles) à l'application de l'un ou l'autre et le recouvrement a montré que l'éthanol semble plus efficace et plus rapide que le $\mathrm{MgCl}_{2}$ (Butler-Struben et al. 2018). De façon surprenante, le froid qui a des effets anesthésiants sur les espèces tempérées comme Sepia officinalis n'est pas efficace chez les espèces tropicales et le recouvrement de comportements normaux est très variable chez les octopus (Agnisola et al. 1996). Sur la base d'observations recensées sur différentes espèces, un référentiel très généraliste sur les effets des produits anesthésiants a été établi. Très sommairement, on peut citer, une diminution des contractions du manteau (= rythme respiratoire ralenti), un relâchement de la pupille, un relâchement des bras, des chromatophores dont le rythme de contraction/relâchement est réduit (il n'y a plus de gradation et d'adaptation des motifs à l'environnement)... et le fait que pincer le manteau ou un bras ne provoque plus de réaction réflexe.

\section{EUTHANASIE}

Il n'y a aucune indication à l'annexe IV de la législation sur un mode opératoire de mise à mort " autorisée " des céphalopodes. En conséquence, quelle que soit la méthode envisagée, toute mise à mort dans le cadre de manipulation à des fins scientifiques implique de prévoir une procédure de " mise à mort dérogatoire " dans une demande d'autorisation de projet au ministère même lorsqu'il s'agit d'un adulte en fin de vie qui présente des manifestations de sénescence. En effet, le cycle biologique des céphalopodes est court et les organismes ne font qu'une seule saison de reproduction (espèces semelpares). Ils meurent, tous et toutes, après la saison de reproduction et de ponte. Ainsi, paradoxalement, la question de l'euthanasie d'un animal qui s'est déjà reproduit se pose en des termes de bien-être pour éviter les désagréments liés à la dégénérescence. Parmi les méthodes appliquées dans la pratique, la surdose de $\mathrm{MgCl}_{2}$ ou d'éthanol est fréquemment utilisée, suivie parfois d'une décérébration. Le refroidissement progressif peut également être utilisé en fonction des espèces, suivi d'une décapitation. Les céphalopodes ont un système circulatoire clos, une " exsanguination " pourrait ainsi être pratiquée directement par décapitation. La mort est observée quand l'animal est devenu complètement blanc et qu'il n'y a plus aucune réaction musculaire (et donc ventilatoire) du manteau.

\section{CAS DES STADES EMBRYONNAIRES}

L'embryon se développe dans l'œuf protégé par une capsule souple individuelle ou " collective " sous forme de cordons. Il se développe sur un sac vitellin par une segmentation discoïdale, semblable au poussin de la poule. Le développement est direct, il n'y a pas de métamorphose ni de stade larvaire planctonique, et au cours de la croissance, le vitellus se résorbe progressivement. A l'éclosion, l'animal ressemble à un adulte, il n'a plus de vitellus et il adopte rapidement le comportement alimentaire de l'adulte. La question était de savoir si les embryons devaient faire l'objet d'une demande d'autorisation de projet ou non. La législation était particulièrement ambiguë à ce sujet. Selon le décret de 2013 : "La présente directive s'applique aux animaux suivants : a) animaux vertébrés non humains vivants, y compris i) les formes larvaires autonomes ; et ii) les formes fotales de mammifères à partir du dernier tiers de leur développement normal ; b) les céphalopodes vivants... ». Il y avait en effet des précisions pour les 
poissons, dont l'autonomie alimentaire est acquise une semaine après éclosion, à résorption du vitellus, mais pour les céphalopodes, seul le terme vivant est utilisé. Cela signifie, si l'on est rigoureux, que même les embryons dans l'œuf sont concernés. Or dans le journal de l'Union européenne du 24 avril 2020 (L129/16) une décision précisant les modalités de prise en compte des céphalopodes a été ajoutée, permettant ainsi de clarifier le décret, à savoir : «1.4. Toutes les espèces céphalopodes doivent être déclarées dans la rubrique "Céphalopodes " à partir du stade où l'animal est capable de s'alimenter seul, c'est-à-dire immédiatement après l'éclosion ". Cela exclut donc les embryons avant éclosion de toute demande d'autorisation de projet. Néanmoins, d'une part, certaines espèces ne se nourrissent pas la première semaine après éclosion, contrairement à ce que laisse suggérer le nouvel avis, mais d'autre part, les embryons sont capables de percevoir et de réagir à des stimuli extérieurs (lumière) bien avant l'éclosion. Cet ajout montre qu'il a été décidé de ne pas en tenir compte et d'appliquer la même règle que pour les oiseaux, à l'éclosion.

\section{CONCLUSION}

Les céphalopodes sont des animaux dont les réactions motrices et sensorielles liées à l'organisation de leur système nerveux suggèrent un ressenti de la douleur. S'ils montrent dans certains cas des capacités d'acclimatation importantes aux changements environnementaux (conditions physico-chimiques fluctuantes), les stimulations entrainant un stress peuvent conduire à des dommages physiologiques irrémédiables. Il convient donc de prendre toutes les précautions nécessaires pour maintenir les animaux dans des conditions optimales de bien-être et de pratiquer toute expérience à des fins scientifiques dans des conditions diminuant (au moins) ou abolissant les réactions vives (= la « douleur ") par l'utilisation d'anesthésiques (chimiques ou non). Ces précautions doivent être prises dès l'éclosion de l'animal, voire avant.

\section{REMERCIEMENTS}

Je remercie Gaëtan Schires (Station biologique de Roscoff-SU) pour son expertise et les discussions fructueuses sur les céphalopodes hébergés dans le cadre de l'expérimentation animale.

\section{BIBLIOGRAPHIE}

- Agnisola C, Castaldo P, Fiorito G. Octopus vulgaris (Mollusca, Cephalopoda) as a model in behavioral pharmacology: A test of handling effects. Physiol Behav 1996; 59:729-733. https://doi. org/10.1016/0031-9384(95)02153-1.

- Andrews PLR, Darmaillacq A-S, Dennison $\mathrm{N}$, et al. The identification and management of pain, suffering and distress in cephalopods, including anaesthesia, analgesia and humane killing. J Exp Mar Biol Ecol. 2013; 447: 46-64. https://doi.org/10.1016/j.jembe. 2013.02.010.

- Butler-Struben HM, Brophy SM, Johnson NA, Crook RJ. In Vivo recording of neural and behavioral correlates of anesthesia induction, reversal, and euthanasia in cephalopod molluscs. Front Physiol; 9: https://doi.org/10.33 89/fphys.2018.00109.

- Crook RJ. Behavioral and neurophysiological evidence suggests affective pain experience in octopus. iScience 2021; 24:102229. https://doi.org/10.1016/ j.isci.2021.102229.

- Crook RJ, Dickson K, Hanlon RT, Walters ET. Nociceptive sensitization reduces predation risk. Curr Biol. 2014; 24:1121-1125. https://doi.org $\angle 10.1016 /$ j.cub.2014.03.043.

- Crook RJ, Hanlon RT, Walters ET. Squid have nociceptors that display widespread long-term sensitization and spontaneous activity after bodily injury. J Neurosci. 2013; 33:10021-10026. https://doi.org/10.1523/JNEURO SCI.0646-13.2013.

- Fiorito G, Affuso A, Anderson DB, et al. Cephalopods in neuroscience: regulations, research and the 3Rs. Invertebr Neurosci. 2014;14:13-36. https://doi.org/10.1007/s10158-013-0 165-x.

- Fiorito G, Affuso A, Basil J, et al. Guidelines for the care and welfare of cephalopods in research -a consensus based on an initiative by CephRes, FELASA and the boyd group. Laboratory Animals 2015; 49: 1-90. https://doi.org/10.1177/0023677215 580006.

- Jiang M, Zhao C, Yan R, et al. Continuous inking affects the biological and biochemical responses of cuttlefish sepia pharaonis. Front Physiol. 2019; 10: https://doi.org/10. 3389/fphys.2019.01429.

- Shomrat T, Turchetti-Maia AL, Stern-Mentch N, et al. The vertical lobe of cephalopods: an attractive brain structure for understanding the evolution of advanced learning and memory systems. J Comp Physiol A. 2015; 201: 947-956 https://doi.org/ 10.1007/s00359-015-1023-6.

-Winlow W, Di Cosmo A. Sentience, pain, and anesthesia in advanced invertebrates. Front Physiol. 2019; 10: 1141.

- Zepeda EA, Veline RJ, Crook RJ. Rapid associative learning and stable long-term memory in the squid euprymna scolopes. Biol Bull-US. 2017; 232:212-218. https://doi.org/ 10.1086 $\angle 693461$. 